\title{
Visible short-pulses generation by nonlinear propagation of necklace beams in capillaries
}

\author{
Aurora Crego $^{1, *}$, Julio San Roman ${ }^{1}$, and Enrique Conejero Jarque ${ }^{1}$ \\ ${ }^{1}$ Grupo de Investigación en Aplicaciones del Láser y Fotónica, Departamento de Física Aplicada, University of Salamanca, E-37008. \\ Salamanca, Spain.
}

\begin{abstract}
We study numerically the propagation of necklace beams through a gas-filled capillary. Ultrashort pulses in the visible (VIS) region can be obtained due to the spectral broadening of these necklace beams. This new source of few-cycle VIS pulses can be generated carrying tens of microjoules of energy using these special beams, being a valuable tool for the improvement of the standard post-compression schemes in terms of spatial stability and output energy.
\end{abstract}

\section{Introduction}

One of the most common post-compression techniques to obtain ultrashort pulses is the nonlinear propagation of a laser pulse as the fundamental mode of a capillary filled with gas [1]. This technique involves the spectral broadening of the laser pulse by self-phase modulation (SPM) followed by a spectral phase compensation to finally obtain the shortest possible output. Although it is possible to obtain few-cycle pulses in the near and mid-infrared [2], the possibility of obtaining short pulses in the visible region of the spectrum $(400-600 \mathrm{~nm})$ with this technique is still very limited. Moreover, to obtain directly an ultra-short pulse at the end of the capillary, one has to be in the anomalous dispersion region, so the pulse self-compresses during its propagation [3].

The fundamental mode of a standard capillary filled with 1 bar of argon (the $\mathrm{EH}_{11}$ hybrid mode) presents a normal response at $800 \mathrm{~nm}$ [4], which prevents the possibility of using this configuration to get self-compression. Higher order spatial modes that present anomalous dispersion [5] are therefore needed. In this work, we explore another possibility which consists in using a particular combination of high spatial modes that results in a linearly polarized beam with a spatial necklace shape [6].

\section{Results}

To study the nonlinear propagation dynamics in capillaries we have developed a $(3+1) \mathrm{D}(\mathrm{r}, \theta, \mathrm{t}, \mathrm{z})$ numerical model based on the nonlinear Schrödinger equation. This model represents the complete spatio-temporal dynamics of a laser pulse propagating in a capillary, and includes dispersion, absorption, self-phase modulation, selfsteepening and ionization.
We have studied the nonlinear propagation of a necklace beam with 6 beads in a capillary. This mode is a composition of the $\mathrm{EH}_{21}$ and $\mathrm{EH}_{41}$ spatial modes [4]. We propagate a 30 -fs pulse with $0,2 \mathrm{~mJ}$ input energy at 800 $\mathrm{nm}$ through a capillary with $150 \mu \mathrm{m}$ core radius filled with argon at 1 bar. We show in Fig. 1 the spatial profile of the necklace beam at the entrance of the capillary. We do not observe any spatial dynamics, like self-focusing, along the propagation through the capillary [7].

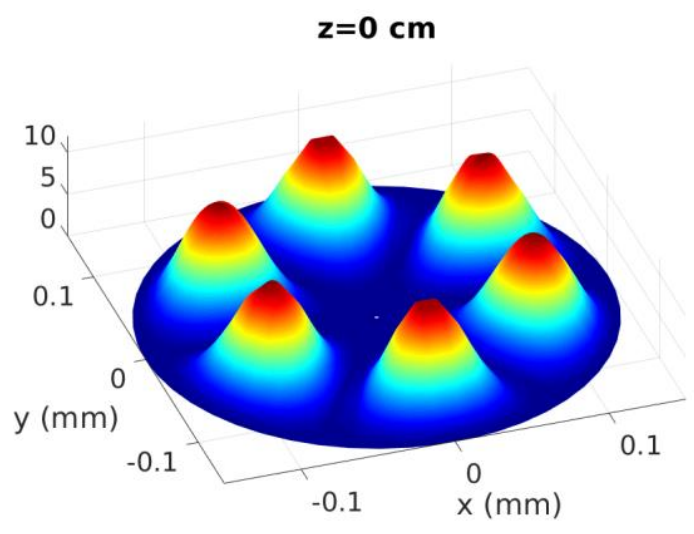

Fig. 1. Transverse profile of the input necklace beam mode with 6 beads.

For the parameters used in the simulation the zerodispersion wavelength (ZDW) is $679 \mathrm{~nm}$, so the input pulse propagates in the anomalous dispersion region. Figure 2 shows the evolution of the temporal and spectral intensity distribution at a point of maximum intensity. The self-phase modulation broadens the spectrum and this dynamic is accompanied by the self-compression towards a solitonic structure at around $70 \mathrm{~cm}$ inside the capillary.

\footnotetext{
* Corresponding author: acrego@usal.es
} 

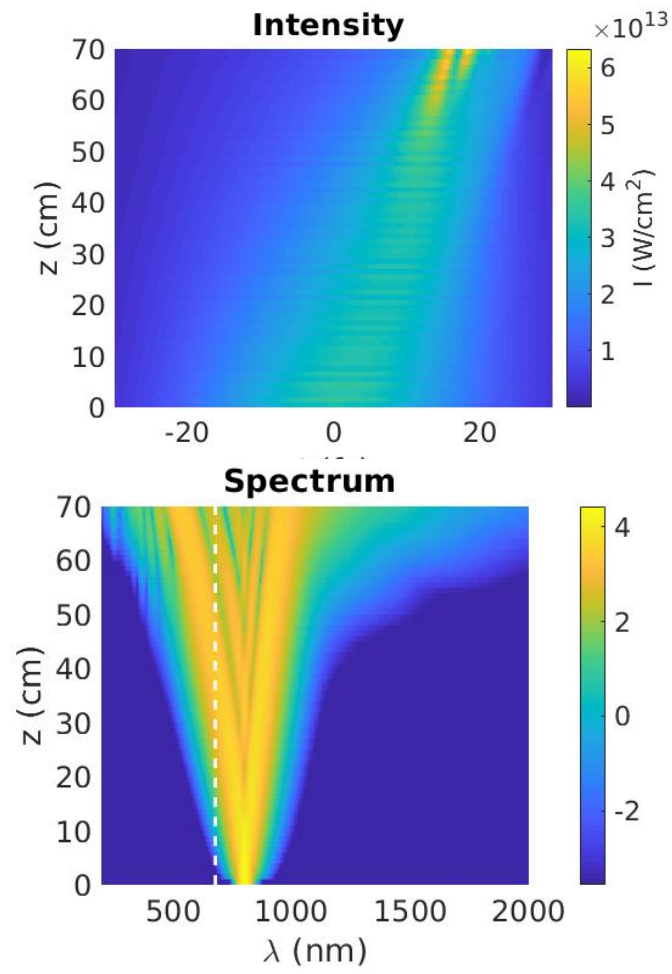

Fig. 2. Evolution of the temporal intensity (top row) and spectral intensity (bottom row, in log scale) distributions at a point of maximum intensity. The white line represents the ZDW at $679 \mathrm{~nm}$.

The most notable feature of the spectrum in Fig. 2 is that it is mainly composed by two spectral bands, one which lies in the anomalous dispersion region and enables the soliton formation, and from the other one, which lies in the normal-dispersion region, we can obtain a short pulse in the visible region of the spectrum.

We have filtered the visible band of the spectrum for a pressure scan (see Fig. 3) to observe the dependence of the dynamics with the pressure. We have compared all the cases at the self-compression distance obtained at 1 bar of pressure $(66 \mathrm{~cm})$. From this comparison we have observed that this visible band suffers a blue-shift until $550 \mathrm{~nm}$ as the pressure increases.

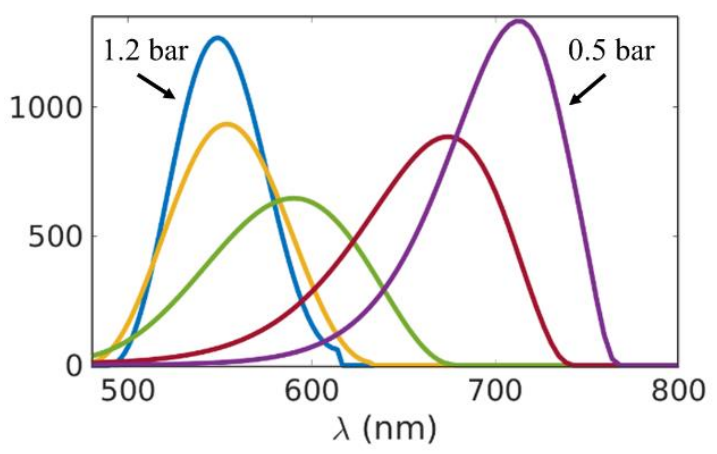

Fig. 3. Filtered visible band of the spectrum at different pressures $(1.2,1,0.8,0.6$ and 0.5 bar from left to right) at 66 $\mathrm{cm}$ inside the capillary.

We have calculated the energy and the full width half maximum (FWHM) duration of the visible band along the propagation. In Fig. 4 we show the dependence with the pressure of the energy and the duration of the pulse and its Fourier limit at $66 \mathrm{~cm}$ inside the capillary. We can observe that the energy values are around $50 \mu \mathrm{J}$ in all cases and the visible pulse durations are less than $10 \mathrm{fs}$. The cases with the lowest energy (0.7-0.8 bar) have the shortest pulse duration reaching $5 \mathrm{fs}$, approximately, and could be post-compressed to a Fourier limit of $3 \mathrm{fs}$.

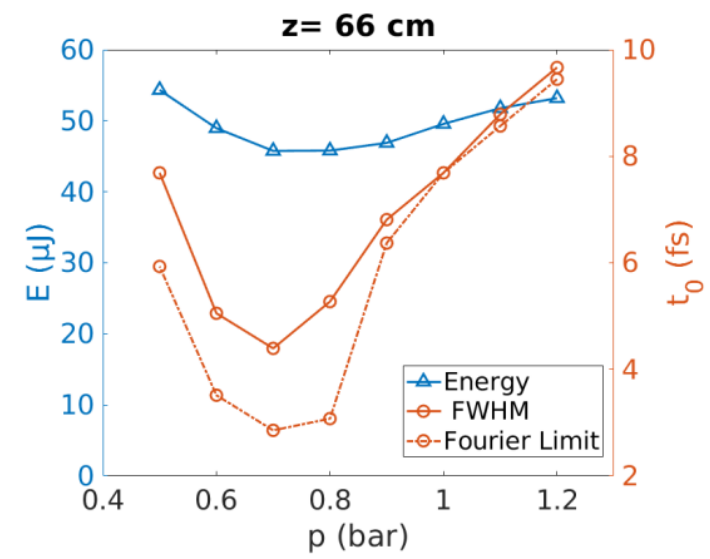

Fig. 4. Energy, FWHM and Fourier limit dependence with the pressure at $66 \mathrm{~cm}$.

We have demonstrated that with the nonlinear propagation of necklace beams in capillaries we can obtain short and energetic pulses in visible wavelengths. We believe that this study paves the way to new short laser pulse sources in the visible region of the spectrum and its subsequent applications.

The authors thank support from Spanish Ministerio de Economía y Competitividad (FIS2016-75652-P) and from Junta de Castilla y León (SA287P18). A.C. is funded by the FPI grant program of Spanish Ministerio de Ciencia e Innovación and European Social Fund (BES-2017-080280).

\section{References}

1. M. Nisoli, S. De Silvestri, O. Svelto, Appl. Phys. Lett. 68, 2793-2795, (1996)

2. E. Conejero Jarque, et al. Sci. Reports 8, 2256, (2018)

3. J. C. Travers, T. F. Grigorova, C. Brahms, F. Belli, Nat. Photonics 13, 547-554 (2019)

4. E. A. J. Marcatili, R. A. Schmeltzer, Bell Labs Technical Journal 43, 1783 (1964)

5. B. A. López-Zubieta, E. Conejero Jarque, I. J. Sola, J. San Roman, OSA Continuum 1, 930-938, (2018)

6. G. Fibich, D. Shpigelman, Physica D 315, 13-32 (2016)

7. A. Crego, E. Conejero Jarque, J. San Roman, Sci Rep 9, 9546 (2019). 